\title{
Selection of W-Pair-Production in DELPHI with Feed-Forward NEURAL NETWORKS
}

\author{
K.-H. Becks, P. Buschmann, J. Drees, U. Müller*, H. Wahlen \\ Fachbereich Physik,Bergische Universität-Gesamthochschule, D-42097 Wuppertal and DELPHI collaboration
}

\begin{abstract}
Since 1998 feed-forward networks have been applied for the separation of hadronic WW-decays from background processes measured by the DELPHI collaboration at different center-of-mass energies of the Large Electron Positron collider at CERN. Prior to the publication of the $189 \mathrm{GeV}$ results (1) intensive studies of systematic effects and uncertainties were performed. The methods and results will be discussed and compared to standard selection procedures.
\end{abstract}

\section{INTRODUCTION}

In 1996 the energy of the Large Electron Positron collider (LEP) at CERN ${ }^{1}$ crossed the threshold energy of 161 $\mathrm{GeV}$ for $\mathrm{W}$-pair-production $e^{+} e^{-} \rightarrow W^{+} W^{-}$. The determination of the $\mathrm{W}$-production cross-section and the direct measurement of the mass of the $\mathrm{W}$-boson allows new tests of the standard model and cross-checks with earlier electroweak measurements.

In 1998 feed-forward neural networks were invented in the selection of hadronic WW-decays $W^{+} W^{-} \rightarrow q \bar{q} q \bar{q}$ measured by the DELPHI ${ }^{2}$ detector at the center-of-mass energy of $189 \mathrm{GeV}$. Due to convincing results this analysis tool has also been used at all higher center-of-mass energies.

\section{SIGNAL AND BACKGROUND PROCESSES}

Standard QCD-events $e^{+} e^{-} \rightarrow Z^{0} / \gamma^{*} \rightarrow q \bar{q}$ are the dominant background to $\mathrm{W}$-pair-production. The second important background is the hadronic decay of the Z-pairproduction $e^{+} e^{-} \rightarrow Z Z \rightarrow q \bar{q} q \bar{q}$ which ends in the same final state with the similar topology like the signal. It is therefore quite important to separate signal from background with high efficiency and purity.

The hadronic WW-decay shows a 4-jet event topology. The former selection process of the "W working group" of the DELPHI collaboration was based upon linear cuts on four variables: the effective center-of-mass energy, the

\footnotetext{
* corresponding author: mueller@whep.uni-wuppertal.de

${ }^{1}$ European Organization for Nuclear Research

2 Detector with Lepton, Photon and Hadron Identifi cation
}

number of jets, their total track multiplicity and the combined variable $D=\frac{E_{\min } \cdot \Theta_{\min }}{E_{\max } \cdot\left(E_{\max }-E_{\min }\right)}$, which consists of the minimum and the maximum jet energy and the minimum angle between two jets $(3,4)$.

\section{FEED-FORWARD ANALYSIS}

The analysis starts with a loose preselection against non-4-jet events and events with reduced center-of-mass energy due to initial state photon radiation.

Then a feed-forward network based upon the JETNET package (2) with a 13 - 7 - 1 architecture is used. So, one hidden layer with 7 nodes and one single output follow the 13 inputs which consist of physical observables which describe the event or jets of WW-decays or background events. All variables have discriminating power known from earlier studies with self-organizing maps (5), the old standard or other high-energy physics analyses.

The training was performed over 1000 cycles with samples consisting of 3500 DELPHI WW- and QCDMonte-Carlo events each generated with ARIADNE (7) or Pythia / JETSET (6). The influence of the DELPHI detector (described in (8)) was simulated with the DELSIM or the FASTSIM package (9).

Tests with an additional ZZ-Monte-Carlo training sample and 3 output nodes gave compatible results but required a higher CPU time. So the easier and faster network was chosen.

After finishing the training, the network output was calculated for independent samples of simulated $W W$, QCD and $Z Z$ events and for real data. 


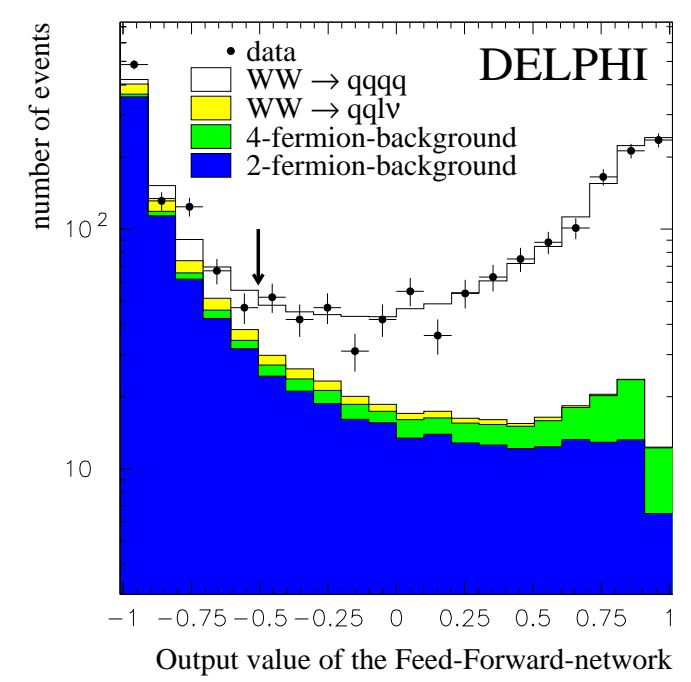

FIGURE 1. neural net output with data-MC agreement

\section{SELECTION QUALITY}

Figure 1 shows the output distribution of the neural network for data and simulated events. The points show the data and the histograms the predicted distributions for the signal and the different backgrounds. Events to the right of the vertical arrow were accepted for the event sample. The data-MC agreement is quite good.

Tab. 1 shows characteristic numbers of the selection quality at $189 \mathrm{GeV}$ for the neural net compared to the standard linear cut analysis. The product of efficiency and purity is a good indicator for the selction quality and the expected statistical error. The neural net analysis shows a clear improvement.

The good data-MC agreement and the improvement in the selection quality were also found at all higher LEP en-

Table 1. selection results of neural net compared to linear cut analysis

\begin{tabular}{|lc|c|c|}
\cline { 3 - 4 } & & NN & cuts \\
\hline signal effi ciency & {$[\%]$} & 88.74 & 85.58 \\
\hline remaining bg & {$[p b]$} & 1.886 & 2.228 \\
\hline selection purity & {$[\%]$} & 77.84 & 74.14 \\
\hline effi ciency $\times$ purity & {$[\%]$} & 69.08 & 63.45 \\
\hline selected events & 1298 & 1342 \\
\hline
\end{tabular}

ergies. So this neural net was chosen for the official DELPHI cross section analysis in the hadronic decay channel.

\section{SYSTEMATIC STUDIES}

This choice made detailed studies of the systematic uncertainties necessary. Two different kinds of tests were performed to determine the systematic errors of the signal efficiency and the remaining background.

First different studies of the network stability were made:

- tests of different network architectures: one or two hidden layers with more or less hidden nodes ;

- variations of important network parameters like learning rate and momentum term around the working point $\left(\eta=0.0025_{-0.0015}^{+0.015}, \alpha=0.56 \pm 0.3\right)$;

- use of different numbers of training events and different training samples .

All these tests gave compatible results within the statistical uncertainties and so no contribution to the systematic errors were assumed.

On the other hand studies were performed with techniques known from the old or other high-energy analyses. Here the neural net was only used as a mathematical function with a fixed training, fixed weights and always the same cut in the output distribution:

- comparison of Monte-Carlo generators with different hadronisation models and different MC parameter settings ;

- data-MC agreement using the technique ${ }^{3}$ of mixed Lorentz-boosted $Z^{0}$ for data taken at the $Z$-resonance at a center-of-mass energy of $91.2 \mathrm{GeV}$;

- smearing of input variables taking detector resolution into account ;

- influence of final state interactions on signal efficiency (Bose-Einstein correlation and color reconnection) .

Each method gave a systematic effect on the selection efficiency and the background. Afterwards the different systematics were combined, trying to take into account correlations between the methods.

\footnotetext{
3 two independent hadronic $Z$ decays were transformed into a pseudo $W$ pair event by applying an appropriate boost to the particles of each $Z$ event
} 


\section{FINAL RESULTS}

Tab. 2 shows the final cross section numbers for 189 $\mathrm{GeV}$ and all higher center-of-mass energies. All results were determined from a binned maximum likelihood fit to the distribution of the neural net output variable above a cut value taking into account the expected background in each bin. The fit also gave the final systematic error for $189 \mathrm{GeV}$ using the uncertainties of efficiency and background as well as other analysis-independent errors (e.g. of the luminosity and the beam energy).The result is:

$$
\sigma_{W^{+} W^{-} \rightarrow q \bar{q} q \bar{q}}=7.36 \pm 0.26(\text { stat }) \pm 0.10(\text { syst }) p b .
$$

The result from the old linear cut analysis was also determined as a comparison:

$$
\sigma_{W^{+} W^{-} \rightarrow q \bar{q} q \bar{q}}=7.56 \pm 0.28(\text { stat }) p b .
$$

Here the systematic error can be expected from the previous years to be compatible to the neural net analysis.

The results agree within the uncertainties and the systematic errors were found to be compatible. But the statistical error of the neural network is lower due to the better selection quality.

\section{SUMMARY}

A feed-forward neural network is successfully used in the DELPHI collaboration to select hadronic WWcandidates and determine the production cross section. The selection quality is clearly improved compared to the previous standard analysis using linear cuts. For this reason the statistical error could be reduced using the neural network. The systematic error was determined performing studies of the network stability and using the neural net as a fixed mathematical function in well known techniques. Finally the systematic error was found to be compatible compared to the standard analysis.

\section{REFERENCES}

1. DELPHI Collaboration,Physics Letters B 479,(2000) 89.

2. Lønnblad,L.,Peterson,C.,Pi,H. and Røgnvaldsson, T., JETNET 3.1 - A Neural Network program for jet discrimination and other High Energy Physics triggering situations Department of Theoretical Physics, University of Lund, Sweden (1994).

3. DELPHI Collaboration,Physics Letters B 397,(1997) 158-170.

4. DELPHI Collaboration,E.Phys.J.C 2,(1998),581.

5. Becks, K.-H., Drees, J., Flagmeyer, U. and Müller, U., Nucl.Instr.Meth. A 426,(1999) 599-604.

6. Sjøstrand, T., Pythia 5.7 and Jetset 7.4 Physics and Manual CERN-TH.7112/93,(1993).

7. Lønnblad, L.,Comp. Phys. Comm. 71,(1992) 15.

8. DELPHI Collaboration,Nucl.Instr.Meth. A 378,(1996) 57.

9. DELPHI Collaboration,DELPHI Note 89-67 and 8968 PROG 142 and 143 (1989).

Table 2. cross section results with statistical errors for different center-ofmass energies (ECM)

\begin{tabular}{|c|c|}
\hline ECM $[\mathrm{GeV}]$ & xsec $[p b]$ \\
\hline 188.6 & $7.36 \pm 0.26$ \\
\hline 191.6 & $7.86 \pm 0.65$ \\
\hline 195.6 & $8.23 \pm 0.39$ \\
\hline 199.5 & $7.90 \pm 0.36$ \\
\hline 201.6 & $7.98 \pm 0.53$ \\
\hline 204.9 & $8.33 \pm 0.40$ \\
\hline 206.8 & $7.74 \pm 0.37$ \\
\hline
\end{tabular}

